\title{
Una mirada cualitativa sobre la autoevaluación institucional desde la praxis universitaria
}

\author{
A qualitative perspective on institutional self-evaluation from university praxis
}

\section{Uma perspectiva qualitativa sobre auto-avaliação institucional da praxis universitária}

\author{
Elvira Patricia Florez-Nisperuza ${ }^{1}$ \\ Alina María Hoyos-Merlano ${ }^{2}$
}

Recibido: junio de 2019

Aceptado: noviembre de 2019

Para citar este artículo: Flórez-Nisperuza, E., P., Hoyos-Merlano, A., M. (2020). Una mirada cualitativa sobre la autoevaluación institucional desde la praxis universitaria. Revista Científica, 37(1), 78-89. Doi: https://doi. org/10.14483/23448350.13645

\section{Resumen}

El presente artículo muestra los resultados parciales de una investigación cualitativa adelantada en el Departamento de Ciencias Naturales de la Universidad de Córdoba, Colombia, relacionada con la caracterización de las prácticas evaluativas desde la reflexión de los procesos de autoevaluación, evaluación institucional y acreditación, con la participación de los estudiantes del $X$ semestre de la Licenciatura en Ciencias Naturales y Educación Ambiental. En esta, a partir de una revisión documental, se logró categorizar a la autoevaluación como el punto de partida y de permanente ejercicio que mantiene la mirada puesta sobre los procesos universitarios que propicia una respuesta para lograr la calidad en el alma mater. Las prácticas evaluativas en general, en las esferas nacional e internacional, develan un grado de aceptación hasta el punto de emitir alrededor de los conceptos, voces dentro del lenguaje pedagógico o del contexto educativo como: proceso, calidad, reflexión, cultura, participación. Lo anterior manifiesta un acercamiento de la atmósfera universitaria con los procesos de evaluación, considerando entonces a la universidad como unidad que genera confianza en el marco de la evaluación, por lo que deberían ser estas las Ilamadas a orientar a las instituciones educativas en Colombia para afrontar las prácticas de cualificación de una forma diferente a como hasta el momento se vive en la escuela, permitiendo que el maestro en formación se conduzca bajo este paradigma que debe ser la cultura de la evaluación. Palabras clave: calidad, evaluación institucional, proceso, reflexión.

\section{Abstract}

This article shows the partial results of a qualitative research carried out in the Department of Natural Sciences of the University of Córdoba, Colombia, related to the characterization of evaluative practices from the reflection of the processes of Self-evaluation, Institutional Evaluation, and Accreditation, with the participation of the students of the $X$ semester and were from a documentary review, it

Universidad de Córdoba. Montería, Colombia, epatriciaflorez@correo.unicordoba.edu.co

Universidad de Córdoba. Montería, Colombia, alinahoyosm@correo.unicordoba.edu.co 
was possible to categorize the self-evaluation as the starting point and permanent exercise to keep the gaze on the university processes and respond with the quality in the alma mater. Evaluative practices in general, nationally and internationally reveal a degree of acceptance to the point of issuing around the concepts, voices within the pedagogical language or the educational context such as: process, quality, reflection, culture, participation; what shows an approach and acceptance of the university atmosphere with the evaluation processes, considering then the university as a unit that generates confidence in the framework of the evaluation, for what should be these the calls then to guide the educational Institutions in Colombia to face these qualification practices in a different way than at present is lived in the school and allowing the teacher information, to conduct himself under this paradigm that must be culture, the culture of the evaluation.

Keywords: quality, institutional evaluation, process, reflection.

\section{Resumo}

Este artigo apresenta os resultados parciais de uma pesquisa qualitativa realizado no Departamento de Ciências Naturais da Universidade de Córdoba, Colombia, relacionados com a caracterização das práticas de avaliação a partir da reflexão dos processos de auto, Avaliação Institucional e Acreditação, com a participação de estudantes $X$ semestre e onde a partir de uma revisão da literatura, foi possível categorizar a auto-avaliação como ponto de partida e exercício permanente, a fim de manter um olho em processos universitários e responder aos mater qualidade alma.

Práticas de avaliação geral a nível nacional e internacional revelam um grau de aceitação ao ponto de emissão em torno dos conceitos, vozes dentro do ensino da língua ou contexto educacional: processos, qualidade, reflexão, cultura, participação; o que mostra uma abordagem e aceitação do ambiente universitário com os processos de avaliação, considerando então a universidade como uma unidade que gera confiança no marco da Avaliação, para quais deveriam ser esses os chamados então para orientar as Instituições educacionais em Colômbia para enfrentar essas práticas de qualificação de uma maneira diferente do que atualmente é vivida na escola e permitir que o professor em formação, se conduza sob este paradigma que deve ser cultura, a cultura da Avaliação.

Palavras-chaves: qualidade, avaliação institucional, processo, reflexão.

\section{Introducción}

La pesquisa realizada muestra el panorama de los países que han venido desarrollando investigaciones alrededor de los procesos de evaluación en las universidades, hallando una mejora en la calidad del servicio, lo que denota aspectos que muestran dónde estuvo y dónde está la clave para que ocurran la sensibilización y acogida de la evaluación. Es así como el presente ejercicio nos conlleva en la lectura de los contextos nacional e internacional, a reaccionar sobre el ejercicio de la autoevaluación, que no debería ser el requisito obligatorio que antecede a la evaluación institucional y a los procesos de acreditación sino más bien, un ejercicio desde la necesidad constante de voltear la mirada de forma permanente y autocrítica sobre sí, entendiendo que las instituciones de educación superior son las germinadoras de un conocimiento universal.

Es por esta razón que sobre esto no se pueden tener miradas esquivas, sino miradas enfocadas a las potencialidades, para que continúen siendo fuertes, sobre las oportunidades de mejora como respuesta a un proceso que se proyecta en el futuro para beneficio de la sociedad. En consecuencia, los líderes en la universidad se constituyen como los primeros en ser llamados al empoderamiento de la autoevaluación a nivel institucional no como requisito, sino como un ejercicio de la lectura holística del escenario universitario en términos globales. Pero, ¿dónde radica la dificultad para que este ejercicio sea asumido de forma rígida y como una tarea asignada desde una cadena de mando, en la que algunas veces el último en el eslabón no sabe, ni conoce lo que se está realizando? ¿Por qué en algunos casos se hace un montaje sobre 
el momento de la autoevaluación y luego este se diluye con el paso del tiempo? En efecto, a pesar de pertenecer a políticas distintas, atendiendo a los usuarios que atiende, y de ahí su naturaleza, la educación superior y la educación escolar tienen puntos en común sobre este aspecto: en ambas se realiza la autoevaluación, pero en la primera, a pesar de todo, se evidencia un carácter más voluntario que en la segunda, como práctica institucional, lo que hace necesario una aproximación teórica orientada hacia procesos de reflexión del profesional de la enseñanza, especialmente al maestro en formación, respecto a la autoevaluación como política de calidad en las escuelas.

En atención a lo anterior, las preocupaciones se orientan hacia la siguiente pregunta de investigación: ¿cuál es la comprensión sobre los conceptos que se tejen alrededor de los procesos de evaluación, en especial de la autoevaluación como requerimiento conceptual y metodológico, que permita en el maestro en formación generar nuevas miradas y actuaciones frente a su ejercicio como docente reflexivo en la acción?

\section{Metodología}

El ejercicio cualitativo se centró en la revisión documental de investigaciones (artículos, proyectos y tesis) alrededor de la autoevaluación con los cuales se establecieron categorías de análisis que permitieron precisar ámbitos poco explorados en este campo, cumpliendo el siguiente procedimiento metodológico con sus respectivos hallazgos:

1. Búsqueda de investigaciones sobre evaluación institucional en la escuela de los que se reportan pocos hallazgos, por lo que se decide ampliar la mirada hacia la educación superior, para una reflexión desde el deber ser en la escuela, en búsqueda de luces que permitan asumir este proceso por parte del maestro en formación.

2. Contraste de las investigaciones sobre evaluación institucional en la educación superior en
Colombia y otros países, en relación con las categorías de autoevaluación-acreditación-calidad educativa y evaluación institucional.

3. Selección de categorías de análisis en atención a la problemática detectada, centrando la atención investigativa con las categorías autoevaluación, evaluación institucional y acreditación.

4. Sistematización de aportes conceptuales y metodológicos para la comprensión de los conceptos de autoevaluación, evaluación institucional y Acreditación, como insumo investigativo de tipo reflexivo con los procesos de evaluación institucional.

5. Análisis descriptivo de los datos tabulados en la herramienta estadística Excel versión 2016, acompañado de un proceso de elaboración conceptual que dio como resultado construcciones investigativas que reafirman el papel de la educación frente al desarrollo de competencias en investigación y, en especial, desde un trabajo de reflexión en la comprensión de los tres conceptos: autoevaluación, evaluación institucional y acreditación.

\section{Resultados}

Con la intención de reflexionar y hallar puntos de encuentro entre la universidad y la escuela desde la experiencia en los procesos de evaluación, permitiendo que el maestro en formación conozca y se sensibilice frente a la importancia de aunar esfuerzos en busca de la calidad educativa, se emprendió una búsqueda de investigaciones alrededor de la evaluación institucional en la educación superior, surgiendo las categorías de análisis autoevaluación, acreditación y evaluación institucional en universidades, inclinadas a evaluar la calidad de los procesos, entendidos estos por Fernández y García (2017) en Cuba, como una parte del sistema de gestión propio de las instituciones de educación superior en lo relativo a la verificación de la correspondencia entre lo planificado y lo logrado, a partir del análisis de sus componentes, 
funciones, procesos y resultados. De este primer rastreo, Ilama la atención cómo a pesar de ser la autoevaluación, la pionera del proceso de evaluación institucional entre los decenios de 1980 y 1990, aún continúa vigente como elemento primordial para que se puedan dar los demás procesos, como un "paso previo", tal como lo plantean en la misma Vigo, Segrea y León (2014) en el artículo "Evaluación Institucional. Una herramienta indispensable en la calidad de los procesos universitarios". Luego, en 1992 aparecen los procesos de acreditación y en Colombia nace la Ley 30, que establece el Sistema Nacional de Acreditación de Instituciones de Educación Superior (SNA).

La figura 1 muestra el panorama de las investigaciones nacionales e internacionales, allí se nota cómo en Colombia el número de investigaciones encontradas son significativas con respeto a otros países y eso tiene una explicación: Colombia se encuentra en la actualidad atravesando uno de los mejores momentos en cuanto a los procesos de acreditación institucional y eso se asocia posiblemente con los incentivos que otorga el Gobierno nacional a las universidades acreditadas en este país y a las estrategias para mejorar la calidad de la educación.
A continuación, se presentan los hallazgos asociados con los conceptos bajo los cuales se inscribe el presente artículo, los cuales emergen producto de las experiencias vividas en cada momento del proceso de evaluación en el contexto universitario, determinándose las categorías y subcategorías que se muestran en la siguiente tabla.

Tabla 1. Categorías y subcategorías frente a la autoevaluación y evaluación institucional.

\begin{tabular}{|c|l|}
\hline $\begin{array}{c}\text { Categoría de } \\
\text { análisis }\end{array}$ & \multicolumn{1}{c|}{ Subcategoría de análisis } \\
\hline \multirow{4}{*}{ Autoevaluación } & $\begin{array}{l}\text { La autoevaluación como proceso } \\
\text { participativo y reflexivo }\end{array}$ \\
\cline { 2 - 3 } & \begin{tabular}{l} 
La autoevaluación como cambio cultural. \\
\cline { 2 - 2 } Evaluación \\
institucional \\
proceso generador de cultura
\end{tabular} \\
\hline \multirow{2}{*}{$\begin{array}{l}\text { La evaluación institucional como } \\
\text { instrumento diagnóstico o espejo de la } \\
\text { realidad. }\end{array}$} \\
\cline { 2 - 2 } & $\begin{array}{l}\text { La evaluación institucional como actividad } \\
\text { La evaluación institucional como reflexión } \\
\text { sobre los cambios y mejoras en la } \\
\text { universidad. }\end{array}$ \\
\hline
\end{tabular}

Fuente: Flórez y Hoyos (2018).

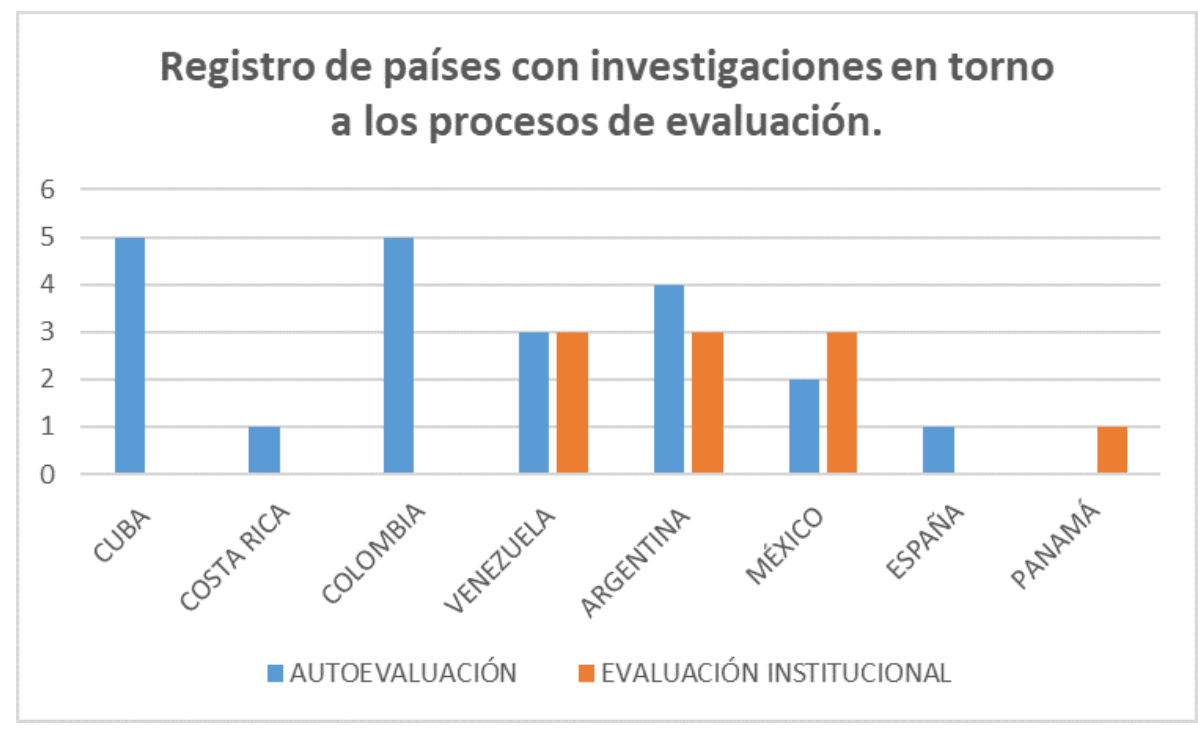

Figura 1. Registro de países con investigaciones en torno a los procesos de evaluación.

Fuente: maestros en formación Universidad de Córdoba, Colombia (2018). 


\section{Autoevaluación}

Esta primera categoría encontrada en el camino de la comprensión como procedimiento conceptual y metodológico en el maestro en formación develó dos líneas de trabajo: la autoevaluación como proceso participativo y reflexivo y la autoevaluación como cambio cultural, conectadas por la búsqueda de la calidad en la educación superior. Ahora bien, para obtener la calidad en la universidad esta debe ser evaluada; se entiende la evaluación de la calidad universitaria como un proceso de autoevaluación participativa —una evaluación interna- cuyos resultados son recomendaciones que deberían articularse "a un plan estratégico de mejora y, por lo tanto, con la capacidad de la unidad evaluada de reelaborar estas propuestas para transformarlas en objetivos o metas estratégicas de su planificación y gestión" tal como lo expresa (Aiello, 2005, p. 196 ) en España en la tesis doctoral titulada "Impacto de la evaluación institucional universitaria en la cultura académica de los profesores".

\section{La autoevaluación como proceso participativo y reflexivo}

En Cuba, Herrera (2010, p.2), en el artículo "la autoevaluación institucional en el contexto de la Universidad Pedagógica: retos y perspectivas", se considera la autoevaluación como un "ejercicio realizado por los propios actores o miembros de la institución que se evalúa, vinculados directamente a los procesos objeto de evaluación". Por tanto, se convierte en uno de los momentos más importantes dado el nivel de integración que genera sobre la comunidad universitaria para lograr un examen hologramático, que permita ver el todo en sus partes y sus partes en el todo, conocer cómo está funcionado la universidad y cómo debería funcionar. Es por ello por lo que para los autores cubanos (López y García, 2014, p. 33) en el artículo "La evaluación y acreditación en la universidad de ciencias pedagógicas. Buenas prácticas en su implantación", la autoevaluación es asumida como un proceso "participativo interno, permanente, de mejora de la calidad, dando lugar a un informe escrito sobre el estado, funcionamiento, procesos, recursos y resultados de la institución, permitiendo la elaboración de un plan de mejora, como antecedente mediático a la evaluación externa". Visto así, la autoevaluación se convierte en un asunto que involucra y compromete a todos los actores que hacen parte del escenario universitario, desde una ruta organizada de planificación, ejecución y control, en la cual desempeña un papel preponderante la Autorevisión o "Autoestudio, que comprende un proceso de reflexión participativa y activa que se plantea a sí misma como objeto de estudio, en donde se explora, analiza, diagnostica, verifica, describe y valora su realidad en cada una de sus estructuras orgánica, académica y administrativa" (Sinaes, 2010,p. 23) en Costa Rica. Desde esa óptica la mirada recae sobre sí misma como mecanismo que implica a la generación de planes que tributen a procesos de calidad continua.

De acuerdo con lo anterior, Dopico (2010, p.730) citado en Vigo, Segrea y León (2014, p.730), en el artículo "Autoevaluación institucional. Una herramienta indispensable en la calidad de los procesos universitarios", indica que la autoevaluación se convierte en un "esfuerzo común por avanzar hacia el reconocimiento mutuo de las decisiones de acreditación [...] lo que implica asegurar y promover estándares de la más alta calidad", el cual también conlleva a hacerla bajo el mismo rigor con el cual será realizada por los evaluadores externos. Tal como lo precisan Vigo, Segrea y León (2014, p.731), cuando expresan que "la autoevaluación debe asumirse con los mismos criterios evaluativos y estándares establecidos en las guías de evaluación de cada país". Así, la autoevaluación ha de permitir una comprensión profunda y oportuna de la institución con la producción de juicios de valor atendiendo a las percepciones de sus miembros y a los criterios interpuestos en la norma. 
Vale la pena resaltar el papel de las universidades cubanas en el interés por realizar investigaciones relacionadas con la autoevaluación institucional; esto responde al porqué ellos ofrecen una educación de alta calidad.

En definitiva, la autoevaluación concebida como un proceso participativo y reflexivo, como un requerimiento conceptual y metodológico, le permite al maestro en formación generar nuevas miradas y actuaciones frente al actuar como docente reflexivo en la acción, en busca de la calidad desde la educación escolar, siendo este el primer escenario en el que se enfoca su experiencia laboral.

\section{La autoevaluación como cambio cultural}

La autoevaluación debe concebirse como un proceso que afecta directamente la posición y el comportamiento individual y colectivo, sobre la forma en que debe asumirse la calidad en todos los campos. De tal manera que esta sea sólidamente implantada y perenne, como un rasgo distintivo que promueva la renovación constante y la consolidación de la universidad. En ese sentido, puede considerarse a la autoevaluación como un "cambio cultural que implica el crecimiento de la conciencia individual y colectiva sobre el sentido de nuestras acciones y la valoración sobre la calidad social de las acciones de investigación, docencia, extensión y acción cultural universitaria" tal como lo plantean en Colombia (Bonilla y Cifuentes, 2010, p. 99) en el artículo "Por el camino de la evaluación institucional en la Universidad Distrital Francisco José de Caldas". En Cuba, Valdés, Nazco y Remedios (2015, p.41) en el artículo "El proceso de autoevaluación en las instituciones universitarias pedagógicas: Una estrategia para su mejora", definen la autoevaluación como "[...] un desarrollo constante o natural que se inserta en la dinámica de la calidad del quehacer diario de la retroalimentación sistemática y la toma de decisiones que favorecen el cumplimiento de la misión en la universidad". En resumidas cuentas, hacer del proceso de autoevaluación una instalación consciente y constante en la educación superior, garantizaría la visibilización contextual del horizonte institucional frente a la formación del tipo de profesional que se desea entregar a los procesos transformacionales de la sociedad, desde la misión, su proyección desde, la visión y la cristalización de los objetivos institucionales en sintonía con las exigencias del mundo globalizante. No obstante, en Colombia, (Hernández Pantoja, 2010, p 17) en el artículo "Calidad y Acreditación en la educación superior: Una aproximación conceptual con mirada crítica", define la autoevaluación como "un proceso que requiere de un modelo que oriente las acciones a realizarse para poder convertirse en un elemento normal y natural", en tanto que no se instala por sí sola, requiere de personas, de un sistema normativo y de un compromiso social, por ser la educación un encargo social.

Por tanto, asumir la autoevaluación como un cambio cultural desde la comprensión de cada uno de los actores, permite que los procesos de florecimiento de esta etapa logren perpetuarse en el escenario en donde se desarrollen, en este caso: educación superior y educación en la escuela, de no ser asumido de esta forma la experiencia de la autoevaluación será un momento efímero.

Con respecto a la dinámica de autoevaluación institucional en las escuelas en Colombia, está orientado por la guía 34, (MEN, 2008) y es vivido como un espacio denominado Semana Institucional, que revisa y pone la lupa desde los distintos agentes evaluadores, sobre el proceso realizado durante el año escolar, arrojando resultados que terminan archivados como insumo de trabajo nuevamente para el próximo año. Asumiendo este proceso, solo como requisito de cumplimiento con las secretarias de educación municipal y departamental, lo que indica que la autoevaluación como cambio cultural aún no se consolida, hasta el punto de ser un campo poco explorado desde el campo investigativo y sobre el cual se debe incursionar en busca de estrategias que fortalezcan dicho proceso en la escuela, siendo esta la base sobre la cual se construye la universidad. 


\section{Evaluación institucional}

En el itinerario de los procesos que le apuntan a la calidad de la educación la evaluación institucional continúa como segundo requisito para aspirar a la acreditación. Se inicia afirmando que la evaluación institucional es un proceso sumamente importante tal como lo indica en Venezuela Perozo (2010, p.22), en la tesis doctoral "La investigación autoevaluación autopoiética. Principio regulador de la evaluación institucional para la consolidación de una cultura evaluativa en las instituciones universitarias venezolanas", al sostener que "La Evaluación Institucional es una herramienta indispensable para el mejoramiento educativo continuo, como fórmula de autorregulación y de rendición de cuentas, en todos los niveles de la educación venezolana, como medio para construir la educación que se desea". Asimismo, parafraseando a Lamarra, AieIlo, \& Grandoli, (2013). en “¿Evaluación para la mejora? La evaluación institucional en las universidades argentinas"; la autoevaluación institucional es comprendida como una estrategia de regulación mediante la cual el Estado reconoce la capacidad de autonomía y autorregulación de las instituciones universitarias para la mejora en el desarrollo de las funciones de la universidad. En este orden, en México la estiman (Juárez, Gonzáles, \& Concha, 2018, p.1457) en el artículo "La evaluación institucional a partir de los indicadores del Sistema de Alerta Temprana", como un "proceso que tiene una importancia especial para la educación superior, porque, directamente, estudia cuestiones vitales como la calidad de la docencia, la investigación y la acción social, la pertinencia académica de sus planes de estudio y la eficiencia administrativa" y herramienta de suma importancia por el cual se pueden estudiar cuestiones vitales como la calidad de la docencia, la investigación y la acción social, la pertinencia académica de sus planes de estudio y la eficiencia administrativa". Es decir, la evaluación institucional pone la lupa sobre todos los frentes de la universidad para mantenerla viva en el tiempo. Por su parte, en España Alfaro y Pérez
(2012, p.26) en el artículo "La evaluación institucional como factor de calidad en la educación superior. Propuesta de un sistema de evaluación", la defienden como "el procedimiento más adecuado para promover la mejora de las instituciones universitarias". Tales posturas mantienen un aliento de reconocimiento de la evaluación como espejo que devela el panorama real de lo que es y, por lo tanto, nos permite aferrarnos a ella para continuar cualificando los procesos universitarios.

A continuación, se presentan las subcategorías explicativas que emergen de las experiencias investigativas alrededor de la evaluación institucional, concebida como proceso, el cual es entendido por la RAE como la "acción de ir hacia adelante", "transcurso del tiempo" y "conjunto de fases sucesivas de un fenómeno natural o de una operación artificial".

\section{La evaluación institucional como un proceso ge- nerador de cultura}

Todo proceso de evaluación institucional debe instalarse en una cultura de la evaluación, así lo comparten Carrasco, González y García (2010, p.34) en su artículo "La implantación de la evaluación institucional en la universidad española" y Ospina (2011,p.66) con su tesis doctoral "Evaluación de la calidad en educación superior. Un estudio de casos en programas académicos", al afirmar que esta "es un proceso que debe desarrollarse continuamente estableciendo cíclicamente tres momentos secuenciales: la autoevaluación o evaluación interna, la evaluación externa y la meta-evaluación o evaluación de la evaluación para el mejoramiento de la calidad en educación de las instituciones". Es este concepto aparece la reflexión sobre el proceso de evaluación; es decir, que se evalúa el mismo acto de la evaluación a través de procesos metaevaluativos.

\section{La evaluación institucional como instrumento diagnóstico o espejo de la realidad}

Adrogué de Deane, Corengia, García de Fanelli y Pita (2014), en el artículo "La investigación en las 
universidades privadas de la Argentina. Cambios tras las políticas de aseguramiento de la calidad y financiamiento competitivo", se conceptualiza a la evaluación institucional como instrumento para detectar puntos fuertes o débiles y áreas a mejorar, en donde se exponen sus objetivos, aportes y condiciones mínimas, así como sus obstáculos principales. Así mismo se suman González (2005, p.67), en el artículo investigación "El impacto del proceso de evaluación y acreditación en las universidades de América", al señalar que la evaluación institucional, "representa un conjunto de procesos mediante el cual la unidad evaluada obtiene información con la que analiza su realidad, identificando sus fortalezas y debilidades para proponer un plan de mejora, fin último de todo proceso de evaluación". Aclara Quintanilla (1998) que el objetivo de la evaluación institucional no es juzgar la actividad, méritos y fallos de los individuos que trabajan en una institución; sino analizar el funcionamiento de la institución como tal, para detectar sus fortalezas y debilidades, así como sus posibilidades de mejora.

\section{La evaluación institucional como actividad compleja}

Cantos y Sánchez, (2010, p.43) en el artículo "Análisis situacional de la evaluación y acreditación de las universidades en Panamá", definen la evaluación institucional como una "actividad compleja que implica un conjunto de procesos que se inicia determinando el sentido de calidad y que surge del análisis de necesidades que posteriormente serán recogidos en la normativa general, partiendo de la priorización". Así mismo, la concibe Gómez (2014) en artículo "El impacto de los mecanismos de evaluación institucional y acreditación de carreras y la calidad del sistema universitario argentino", cuando cataloga a la evaluación institucional como una actividad compleja que debe garantizar, en primera instancia, el marco normativo y las características de evaluación, analizando logros y dificultades en el cumplimiento de sus funciones; así como sugerir medidas para su mejoramiento. Farias (2010), por su parte, en el artículo "Modelo de evaluación institucional para la Universidad Nacional Experimental de Guayana" (UNEG), Venezuela, presenta a la evaluación institucional como “un proceso sistémico y sistemático de la gestión institucional que contribuye a la medición, la valoración y la retroalimentación para la toma de decisiones, así como para el mejoramiento continuo y la transformación institucional".

Los autores anteriores entonces califican a la evaluación institucional como una actividad compleja por todo el rigor que encierra este proceso, fijando en primera instancia el fin último del proceso evaluativo "la calidad" y determinando lo fundamental para lograr el tránsito de las debilidades a las fortalezas, de la oscuridad del problema a la toma de decisiones, que permitan un panorama seguro y de confianza para el usuario del servicio educativo y de todas las personas involucradas directa e indirectamente con la universidad; esto, bajo la tutela de la norma vigente a través de un proceso ordenado, metódico pertinente legal y alcanzable en el tiempo, tal como se expresa en el siguiente concepto:

La Evaluación Institucional se concibe como un proceso que genera resultados a corto y mediano plazo, cuyo sentido es emitir juicios de valor, documentados e informados, con base en mediciones, análisis, diagnósticos y estudios, útiles para la toma de decisiones. Esta perspectiva resalta el carácter estratégico de la evaluación como un instrumento al servicio de la planeación y la formulación de decisiones institucionales. (Dirección General de Evaluación Institucional normado por la UNAM, (s.f.) citado por Montes, Ceniceros y Aldama (2016).

De acuerdo con lo anterior, emergen factores que en muchos casos permiten reconocer a la evaluación institucional como una carga, dado a la cultura que se debe tener con relación a lo que no se venía haciendo, antes de abrirle camino a esta etapa y es el hecho de recoger, interpretar, 
sistematizar, llevar estadísticas, documentar, soportar con evidencias, diseñar indicadores, hacer comparativos, emitir juicios de valor que permitan contrastar, denotar los avances frente a las metas propuestas y continuar realizando ajustes, estableciendo entonces a la evaluación institucional como un proceso continuo. Tal como coincide en algunos aspectos Cardona, y Perales (2010) en el artículo "Evaluación de la calidad de los programas de maestría y doctorado en Iberoamérica".

Bezies (s.f. p 15) en el artículo "La evaluación institucional: Universidad Autónoma del Estado de Hidalgo", brinda argumentos que respaldan el nivel de rigurosidad que guarda la evaluación institucional, al concebirla como "un proceso que busca ajustar la realidad institucional involucrando de manera integral la visión, misión, objetivos, procesos, logros, deficiencias, identificar problemas, jerarquizar causas y consecuencias, generar conocimientos y reflexiones para proponer soluciones, correctivos, cambios y reajustes oportunos con el propósito de incidir en el mejoramiento de la calidad". Es decir, se debe alinear el horizonte institucional de tal manera que lo que está escrito se vuelva visible y determine el rumbo de cualificación al que se desea llegar; tal como lo proponen en España Romero y Triviño (2009,p.190), en el artículo "La evaluación en la universidad: un proceso de aprendizaje en las organizaciones universitarias", cuando expresan que "se deben proponer con fundamento teórico, acciones necesarias para reconducir las actuaciones hacia la mejora y abrir vías de investigación futuras sobre las demandas y satisfacción del cliente, motivación e implicación de las personas y satisfacción de la comunidad en general".

De acuerdo con lo dicho, y reconociendo la diversidad de innovaciones en las cuales están inmersos los que forman parte de la universidad, se deben realizar procesos que permitan asumir con serenidad y responsabilidad esa actividad compleja, dando lugar a nuevas categorías que posibilitan procesos de reflexión sobre el concepto en cuestión.

\section{La evaluación institucional como reflexión sobre los cambios y mejoras en la universidad}

Martínez (2016,p.15) en la investigación "Autoevaluación institucional, el papel de las variables sociodemográficas en la percepción de los docentes en México" expresa que "la Evaluación Institucional es un proceso de análisis estructurado y reflexivo del sumario institucional en la que se involucran los actores y el contexto para reconocer los procesos y productos, a través de un diálogo comprensivo con la intención de emitir juicios de valor, que sirvan de base para tomar decisiones que permitan la mejora institucional". Indiscutiblemente, los procesos que se delinean alrededor de la educación deben ser mediados por espacios de reflexión dado al objeto que está en juego: la calidad de los procesos al interior de la universidad, en donde lo que se ofrece es un servicio educativo de formación profesional, el cual debe ser cada vez más refinado en todos los aspectos. Así es como (Aristimuño 2015, p.67), en la tesis doctoral "Desarrollo de un modelo integral de evaluación para instituciones de educación superior y análisis de su impacto en la planificación y gestión institucional", señala que la evaluación institucional no debe ser considerada solo como elemento de rendición de cuentas, sino que su fin último es generar una reflexión sobre los cambios y mejoras para las organizaciones universitarias.

Solo cuando se concibe la evaluación institucional como un proceso de reflexión sobre la vida universitaria, se logra transformar la universidad sin presión y de forma consciente, realizándola por la necesidad de refinar los procesos que la enaltezcan en todos los ámbitos. El concepto anterior dista de la posición de Ariana De Vincenzi (2009, p.91), en el artículo "Evaluación institucional y mejoramiento de la calidad educativa en tres universidades privadas argentinas", en el que se considera que "la evaluación institucional y la autoevaluación son muy útiles, pero no es algo que se tenga como una prioridad importante, salvo que haya un requerimiento desde el Estado". Es decir, 
que el autor no la asume como un proceso al cual están Ilamadas todas las universidades de forma voluntaria, sino que debe realizarse solo si tiene un carácter ineludible.

En España, González, García y Triviño (2014,p.19) en el artículo "La evaluación institucional en la universidad: percepción del profesorado y del personal de Administración y Servicios" indican que la evaluación institucional responden a un proceso de aprendizaje representado como "un conjunto de procedimientos y estrategias encaminados al compromiso con la mejora continua, la sistematización en la resolución de problemas y el fortalecimiento del personal", lo cual indica que la evaluación institucional no solo le deja un legado a la universidad, sino a los miembros que en ella participan.

\section{Conclusiones}

En atención a que el artículo se hizo con los estudiantes de $X$ semestre de la Licenciatura en Ciencias Naturales y Educación Ambiental a partir de una revisión documental, ante la mirada cualitativa sobre la autoevaluación institucional desde la praxis, se resalta que lograron tener una perspectiva amplia acerca de la comprensión de la autoevaluación a partir de un proceso de investigación cualitativa, actuando de participante activos en este primer recorrido investigativo y precisando los siguientes aspectos:

Se permitió comprender que, en medio de los procesos de evaluación, triunfa el lenguaje pedagógico de la reflexión, la cultura y los procesos cíclicos que deben permanecer perennes, como es el caso de la autoevaluación.

Se consiguió ver a la universidad como pionera del proceso de autoevaluación, que le sirve de espejo a las instituciones educativas para continuar hacia un modelo de consecución de la calidad educativa en todos los ámbitos.

Se logró asumir un juicio de valor positivo frente a la autoevaluación, recobrando las bondades que muchos desprecian en la escuela; hecho que se convierte en ganancia para el maestro en formación, como representante fiel y esperado en las instituciones educativas para la renovación y resignificación de las prácticas.

\section{Agradecimientos}

A la Universidad de Córdoba por avalar desde el centro de investigaciones el proyecto de investigación "Caracterización de las prácticas evaluativas de las Instituciones Educativas del departamento de Córdoba como campo de formación académico e investigativo en el estudiante de la Licenciatura de Ciencias Naturales y Educación Ambiental de la Universidad de Córdoba". Así mismo, al programa de Licenciatura en Ciencias Naturales y Educación Ambiental, al grupo de investigación GICNEA y a los estudiantes y docentes del diplomado "Evaluación formativa como estrategia comprensiva. Perspectivas didácticas contemporáneas" 2018-I, por involucrarse en la organización y búsqueda de información en torno a la comprensión de la evaluación institucional.

\section{Referencias}

Addine Fernández, F.; García Batista, G. (2017). Evaluación de la calidad en las instituciones de educación superior cubanas: un reto a certificar. Congreso Universidad, 6(1).

Adrogué de Deane, C.; Corengia, Á.; García de Fanelli, A.; Pita, M. (2014). La investigación en las universidades privadas de la Argentina. Cambios tras las políticas de aseguramiento de la calidad y financiamiento competitivo. REICE. Revista Iberoamericana sobre Calidad, Eficacia y Cambio en Educación, 12(3), 73-91.

Aiello, M. (2005). El impacto de la evaluación institucional universitaria en la cultura académica de los profesores. Tesis doctoral. Universidad de Barcelona, España.

Alfaro, F. A.; Pérez, D. (2012). La evaluación institucional como factor de calidad en la Educación Superior: propuesta de un sistema de 
evaluación. Congreso Virtual Internacional sobre Innovación Pedagógica y Praxis Educativa. Sevilla- España.

Aristimuño, M. (2015). Desarrollo de un modelo integral de evaluación para instituciones de educación superior y análisis de su impacto en la planificación y gestión institucional. Tesis doctoral. Universidad Politécnica de Madrid, España.

Bezies, P. (s. f.). La evaluación institucional: Universidad Autónoma del Estado de Hidalgo. http://intranet.uaeh.edu.mx/evaluacion/documentos/ev institucional.doc

Bonilla Estévez, M.; Cifuentes Traslaviña, M. T. (2010). Por el camino de la evaluación institucional en la Universidad Distrital Francisco José de Caldas. Enunciación, 15(2), 95-102. https://doi.org/10.14483/22486798.3162

Cantos, M.; Sánchez, L. C. (2010). Análisis situacional de la evaluación y acreditación de las universidades en Panamá. In Universidad 2010. Congreso Internacional de la Educación Superior, (6to. Congreso: 2010: Palacio de las Convenciones, Cuba) (No. 378 378). e-libro, Corp.

Cardona, V. E. C.; Perales, F. J. M. (2010). Evaluación de la calidad de los programas de maestría y doctorado en Iberoamérica. Revista Digital Universitaria, 11 (5), 1067-6079. http://www.ru.tic. unam.mx/bitstream/handle/123456789/1621/ art45 2010.pdf? sequence $=1$ \&isAllowed $=y$

Carrasco, M. J.; González, S.; García, M. (2010). La implantación de la evaluación institucional en la universidad española. Proyecto de investigación. Universidad de Huelva, Facultad de C.C. de la Educación, España.

De Vincenzi, A. (2013). Evaluación institucional y mejoramiento de la calidad educativa en tres universidades privadas argentinas. Revista Iberoamericana de Educación superior, 9(4), 76-94. https://doi.org/10.22201/ iisue.20072872e.2013.9.85

Farias, L. (2010). Modelo de evaluación institucional para la Universidad Nacional Experimental de Guayana (UNEG). Revista Kaleidoscopio, 7(13), 40-59.
Fernández-Lamarra, A.; Grandoli, M. (2013). ¿Evaluación para la mejora? La evaluación institucional en las universidades argentinas. Nuevo Pensamiento y Sociedad. Aportes de Posgrados Estudios de Política y Administración de la Educación, 2.

Gómez, J. C., (2014). El impacto de los mecanismos de evaluación institucional y acreditación de carreras y la calidad del sistema universitario argentino. 2250-8465. Buenos Aires. http:// jornadassociologia.fahce.unlp.edu.ar

González, L. E. (2005). El impacto del proceso de evaluación y acreditación en las universidades de América Latina. Varios, El proceso de acreditación en el desarrollo de las universidades. Santiago CINDA.

González-Losada, S.; García-Rodríguez, M. P.; Triviño-García, M. A. (2014). La evaluación institucional en la universidad: percepción del profesorado y del personal de administración y servicios. REDU. Revista de Docencia Universitaria, 12(4), 17-37. https://doi.org/10.4995/ redu. 2014.5613

Hernández, P. (2010). Calidad y acreditación en la educación superior: Una aproximación conceptual con mirada crítica. Revista Criterios, 26, 5-10.

Herrera Serrano, D.; Sánchez Cabrera, S. (2010). La autoevaluación institucional en el contexto de la universidad pedagógica: retos y perspectivas. In Universidad 2010. Congreso Internacional de la Educación Superior, (6to. Congreso: 2010: Palacio de las Convenciones, Cuba) (No. 378 378). e-libro, Corp.

Juárez, Á. I. R.; Gonzáles, A. K. L.; Concha, I. A. C. (2018). Evaluación institucional a partir de los indicadores del Sistema de Alerta Temprana. Debates en Evaluación y Currículo. Congreso Internacional de educación. México.

Lamarra, N. F.; Aiello, M.; Grandoli, M. E. (2013). ¿Evaluación para la mejora? La evaluación institucional en las Universidades Argentinas. Estudios de Política y Administración de la Educación, 26, 27-46. 
Londoño, O. L.; Maldonado, L.; Calderón, L. (2014). Guías para construir estados del arte. Bogotá: International Corporation of Networks of Knowledge.

López, A.; Garcia, M. (2014). La evaluación y acreditación en la universidad de ciencias pedagógicas. Buenas prácticas en su implantación. Atenas, 27(3), 28-41.

Martínez, V. M. (2016). Autoevaluación institucional, el papel de las variables sociodemográficas en la percepción de los docentes en México. En Evaluación institucional, práctica educativa y desarrollo académico (capítulo II, p. 15).

Ministerio de Educación Nacional (1992). Ley 30. Organización del servicio público de la educación superior. Art.55. La autoevaluación institucional en Colombia.

Ministerio de Educación Nacional (2008). Guía para el mejoramiento institucional-MEN n. ${ }^{\circ}$ 34. https://www.mineducacion.gov.co/1759/w3-article-177745. html? noredirect $=1$

Montes, R. F. V., Ceniceros, C. D. I., y Aldama, H. C. R. (2016). Evaluación institucional: práctica educativa y desarrollo académico. Red Durango de Investigadores Educativos A. C. México

Ospina, R. (2011). Tesis doctoral de evaluación de la calidad en educación superior. Un estudio de casos en programas académicos. Universidad Complutense de Madrid, España.

Perozo, L. (2010). Autoevaluación autopoiética. Principio regulador de la evaluación institucional para la consolidación de una cultura evaluativa en las Instituciones Universitarias venezolanas. Tesis doctoral. Universidad de Carabobo, Venezuela.

Quintanilla, M. (1998). En pos de la calidad: notas sobre una nueva frontera para el Sistema Universitario Español. Revista de Educación, 315,85-95.

Romero, G.; Triviño, G, (2009). La evaluación en la universidad: un proceso de aprendizaje en las organizaciones universitarias. Universidad de Huelva. Revista de la UHU XXI: Revista de Educación XXI, V. 08.

Sinaes (2010). Manual de acreditación oficial de carreras de grado. San José, Costa Rica.

Valdés, M.; Nazco, A; Remedios, M. (2015). El proceso de autoevaluación en las instituciones universitarias pedagógicas: Una estrategia para su mejora. Gaceta Médica Espirituana, 17(2), 39-49.

Vigo-Cuza, P.; Segrea-González, J.; León-Sánchez, B.; López-Otero, T.; Pons-Mena, J.; León-Sánchez, C. (2014). Autoevaluación institucional. Una herramienta indispensable en la calidad de los procesos universitarios. MediSur, 12(5), 727-735. 